\title{
Isolation of Complementary DNA for Bullous Pemphigoid Antigen by Use of Patients' Autoantibodies
}

\author{
John R. Stanley, Toshihiro Tanaka, Stephan Mueller, Vera Klaus-Kovtun, and Dennis Roop \\ Dermatology Branch and Laboratory of Cellular Carcinogenesis and Tumor Promotion, National Cancer Institute, \\ National Institutes of Health, Bethesda, Maryland 20892
}

\begin{abstract}
Autoantibodies from bullous pemphigoid (BP) patients define a 230-kD protein found in the basement membrane of stratified squamous epithelia. The purpose of this study was to isolate and characterize a cDNA clone with coding sequences for BP antigen. $P$ oly $\left(\mathrm{A}^{+}\right)$RNA derived from total RNA of cultured keratinocytes was used, with oligo-dT priming, to construct a cDNA library in the $\lambda$ gt11 expression vector, which was screened by the immunoperoxidase method with one BP serum. One darkly stained clone, called here the BP clone, was further characterized. 9 of $9 \mathrm{BP}$ sera, but none of 6 normal and 11 pemphigus sera, bound the plaques of this BP clone. Furthermore, BP IgG affinity purified on plaques of this clone, but not unrelated clones, bound the epidermal basement membrane by immunofluorescence and immunoprecipitated the 230-kD BP antigen from extracts of cultured keratinocytes. Eco RI digestion of the BP clone's cDNA insert demonstrated a 680and 1,500-bp fragment. Northern blots of total keratinocyte RNA showed that complementary riboprobes transcribed from both fragments hybridized to a 9-kb RNA. Dideoxy DNA sequencing from the $5^{\prime}$ end of the BP cDNA demonstrated a 1,992-bp open reading frame, encoding a peptide of $76 \mathrm{kD}$. This BP cDNA clone will be valuable for understanding the protein structure, expression, and gene organization of BP antigen.
\end{abstract}

\section{Introduction}

Bullous pemphigoid (BP) ${ }^{1}$ is an autoimmune blistering disease in which autoantibodies bind the epidermal basement membrane zone (BMZ) and, probably as a result of this binding, subepidermal blisters develop $(1,2)$. These autoantibodies are found in the BMZ of the patients' skin and in their sera. By indirect immunofluorescence these BP autoantibodies bind the BMZ of normal stratified squamous epithelia, not only in human tissue but in the tissue of most vertebrates (3). Thus, these autoantibodies define a molecule found in the normal

Address correspondence and reprint requests to Dr. John R. Stanley, Building 10, Room 12N238, National Institutes of Health, Bethesda, MD 20892.

Received for publication 17 March 1988 and in revised form 23 June 1988.

1. Abbreviations used in this paper: BMZ, basement membrane zone; BP, bullous pemphigoid; IPTG, isopropyl $\beta$-thiogalactopyranoside; LB, Luria-Bertani medium; ORF, open reading frame.

The Journal of Clinical Investigation, Inc.

Volume 82, December 1988, 1864-1870
BMZ of stratified squamous epithelia, such as skin and mucous membranes.

Recent immunoelectron microscopic studies have demonstrated that BP autoantibodies bind to both the extracellular and intracellular parts of the hemidesmosomes of the basal keratinocytes (4-6). Because BP antigen is only found associated with basal keratinocytes, it is a useful marker for this relatively undifferentiated basal cell (7). In addition, basal cells, but not more differentiated keratinocytes, synthesize BP antigen (8).

With the above data in mind, characterization of BP antigen would be useful in furthering our understanding of several aspects of skin and mucous membrane biology, such as: $(a)$ the normal BMZ of stratified squamous epithelia; $(b)$ the structure and function of normal hemidesmosomes; $(c)$ the regulation of synthesis of differentiation molecules in the epidermis; and $(d)$ the pathophysiology not only of BP, but also, perhaps, of certain hereditary diseases such as junctional epidermolysis bullosa, in which blisters occur in the BMZ of stratified squamous epithelia and there are abnormalities of the hemidesmosomes (9).

Thus far characterization of BP antigen has been carried out mostly by immunochemical means. Recent studies have shown that although there may be some heterogeneity of the BP antigen, autoantibodies from almost all patients bind an $\sim 230-\mathrm{kD}$ protein as determined either by immunoprecipitation of extracts of cultured keratinocytes or by immunoblotting of extracts of normal human epidermis (10-13). For example, in our laboratory sera from 26 of 27 BP patients immunoprecipitate a $230-\mathrm{kD}$ polypeptide from extracts of cultured keratinocytes, whereas none of 59 diseased and normal sera controls bind this molecule (12). This finding was confirmed by another laboratory (5). The specificity of these immunochemical studies have established this $230-\mathrm{kD}$ protein as the major BP antigen.

Another useful approach to further characterize BP antigen and to elucidate its amino acid sequence, protein structure, control of its expression, and the organization of its gene would be by molecular biologic techniques. A useful first step in this direction would be the isolation of a cDNA fragment or probe that contains coding sequences for BP antigen. The purpose of this study was to isolate and characterize such a cDNA clone using sera from BP patients.

\section{Methods}

Sera. One BP serum that gave a particularly clean immunoprecipitation and immunoblotting of the $230-\mathrm{kD}$ BP antigen was originally used to screen the cDNA expression library. Because human sera contain antibodies to keratins (14), this serum was preabsorbed with keratin extracted from cultured ME180 cells as follows. Twelve $150-\mathrm{mm}$ culture dishes of confluent cells were extracted with $12 \mathrm{ml}$ of $0.6 \mathrm{M}$ $\mathrm{KCl}, 0.1 \mathrm{M}$ EDTA, $1 \%$ Triton X-100 in Dulbecco's Ca- and Mg-free 
PBS with $1 \mathrm{mM}$ PMSF. The pellet from centrifugation at 15,000 rpm in an SS34 rotor (DuPont Instruments-Sorvall Biomedical Div., Wilmington, DE) was washed with PBS then solubilized with $4 \mathrm{ml}$ of 8-M urea and $1 \mathrm{M}$ 2-mercaptoethanol in $50 \mathrm{mM}$ Tris- $\mathrm{HCl}, \mathrm{pH}$ 9.0. The supernatant, after centrifugation at $2,000 \mathrm{rpm}$, was dialyzed against $0.15 \mathrm{M} \mathrm{NaCl}$ in $10 \mathrm{mM}$ Tris- $\mathrm{HCl}, \mathrm{pH} 7.4$, which resulted in precipitation of keratins. The keratin pellet was collected by centrifugation at $100,000 \mathrm{~g}$ for $30 \mathrm{~min} .1 .5 \mathrm{ml}$ of the BP serum was absorbed three times with $\sim 5 \mathrm{mg}$ (each absorption) of keratin at room temperature for $1 \mathrm{~h}$. Indirect immunofluorescence on monkey esophagus indicated no difference in the anti-BMZ titer of the serum before and after absorption.

As specificity controls for the staining of the putative BP fusion protein we used an additional eight BP sera, each of which was known to immunoprecipitate the $230-\mathrm{kD}$ BP antigen, and as negative controls we used sera from seven patients with pemphigus vulgaris, four patients with pemphigus foliaceus, and six normal individuals. These BP and control sera were not preabsorbed with keratin.

Human keratinocyte cell culture. Keratinocytes from neonatal foreskin were cultured on irradiated 3T3 cells as previously reported (15) and used in the first passage. Cells grown under these conditions have been shown to synthesize BP antigen (15).

Construction of $\lambda$ gt 11 library. In order to find a cDNA clone with coding sequences for BP antigen, we constructed a cDNA expression library in $\lambda g t 11$ as originally described by Young and Davis (16). Total RNA was extracted from each of eight 150 -mm dishes of just confluent human keratinocytes with $5 \mathrm{ml}$ of 4-M guanidine isothiocyanate, $0.5 \%$ sodium lauroyl sarcosine, $0.1 \mathrm{M}$ 2-mercaptoethanol in $25 \mathrm{mM}$ sodium citrate buffer, $\mathrm{pH}$ 7. The extracted RNA was pelleted through a 5.7-M $\mathrm{CsCl}_{2}$ cushion (17) in an SW 41 rotor (Beckman Instruments, Inc., Palo Alto, CA) at $30,000 \mathrm{rpm}$ for $24 \mathrm{~h}$. The resultant RNA pellet was solubilized in autoclaved, $0.1 \%$ diethyl pyrocarbonate-treated deionized water $\left(\mathrm{H}_{2} \mathrm{O}\right.$ [dep]), ethanol-precipitated (18), then redissolved in $\mathrm{H}_{2} \mathrm{O}$ (dep). The yield of RNA was $\sim 0.73 \mathrm{mg} / 150-\mathrm{mm}$ dish of cells. Poly $\left(\mathrm{A}^{+}\right)$RNA was isolated from total RNA by oligo-dT-cellulose (Pharmacia Fine Chemicals, Piscataway, NJ) chromatography (18). The yield of poly $\left(\mathrm{A}^{+}\right)$RNA was $\sim 1.3 \%$ of total RNA.

The quality of the keratinocyte poly $\left(\mathrm{A}^{+}\right) \mathrm{RNA}$ was tested by in vitro translation with a rabbit reticulocyte lysate kit (DuPont New England Nuclear, Wilmington, DE). When compared with the same amount of control yeast mRNA (as judged by $\mathrm{OD}_{260}$ determination and adjusted for the fact that the control yeast RNA contained one-half tRNA), the keratinocyte poly $\left(\mathrm{A}^{+}\right)$RNA directed $>20 \%$ more $\left[{ }^{35} \mathrm{~S}\right]-$ methionine incorporation into TCA-precipitable protein. In addition, SDS-PAGE separation of the translated protein products demonstrated well-defined bands up to $\sim 200 \mathrm{kD}$.

$10 \mu \mathrm{g}$ of the keratinocyte poly $\left(\mathrm{A}^{+}\right) \mathrm{RNA}$ was used to construct a cDNA $\lambda$ gt 11 library at Clontech Laboratories (Palo Alto, CA) as follows: $(a)$ cDNA was made with an oligo-dT primer by the method of Gubler and Hoffman (19); (b) internal Eco RI sites were methylated; (c) Eco RI linkers were ligated to cDNA; $(d)$ cDNA was fractionated to $>500 \mathrm{bp}$ on Sepharose 4B; and (e) cDNA was cloned into the $\lambda \mathrm{gt} 11$ Eco RI site. Average cDNA insert size in the library was about $1 \mathrm{~kb}$ (range 0.5-3.8 kb). Further characterization of this library is discussed in Results.

Screening of $\lambda g t 11$ expression library. Screening of the expression library for clones expressing fusion proteins that bound BP sera was done as described (20) with the following modifications. Y1090 Escherichia coli (E. coli) were grown overnight in $50 \mathrm{ml}$ Luria-Bertami medium (LB) with $0.2 \%$ maltose, $10 \mathrm{mM} \mathrm{MgSO}_{4}$, and $100 \mu \mathrm{g} / \mathrm{ml}$ ampicillin. The cells were suspended in $20 \mathrm{ml} 10 \mathrm{mM} \mathrm{MgSO}_{4}$ and stored at $4^{\circ} \mathrm{C}$ for up to $1 \mathrm{wk} .100 \mu \mathrm{l}$ of phage diluted in $0.1 \mathrm{M} \mathrm{NaCl}, 10$ $\mathrm{mM} \mathrm{MgSO}_{4}, 0.01 \%$ gelatin in $50 \mathrm{mM}$ Tris- $\mathrm{HCl}, \mathrm{pH} 7.5$ (TMG), $100 \mu \mathrm{l}$ of Y 1090 cells, and $100 \mu \mathrm{l}$ of TMG were incubated for $20 \mathrm{~min}$ at $37^{\circ} \mathrm{C}$. This mixture was then plated in $7.5 \mathrm{ml}$ top agarose containing $10 \mathrm{mM}$ $\mathrm{MgSO}_{4}$ on $150-\mathrm{mm} \mathrm{LB}$-agar plates (also containing $10 \mathrm{mM} \mathrm{MgSO}_{4}$ ). As described (20), nitrocellulose filters saturated with isopropyl $\beta$ thiogalactopyranoside (IPTG) were incubated on phage plaques overnight to induce synthesis of fusion protein. These nitrocellulose filters were then used for immunoperoxidase staining with serum to localize plaques that produce fusion proteins that bind particular sera (11). Sera were diluted $1: 100$ in $3 \% \mathrm{BSA}$ in $0.15 \mathrm{M} \mathrm{NaCl}, 0.5 \% \mathrm{NP}-40$ in 10 $\mathrm{mM}$ Tris- $\mathrm{HCl}, \mathrm{pH}$ 7.4. The diluted sera were absorbed twice with $1 / 50$ their volume of $E$. coli lysate (Bio-Rad Laboratories, Richmond, CA).

Affinity purification of antibodies on $\lambda$ gt 11 plaque protein. To obtain the affinity-purified BP IgG that bound protein produced by the purified BP $\lambda g t 11$ clone, we plated the isolated clone at a density (just short of confluence) of 1,000-4,000 plaques/150-mm plate. Fusion protein synthesis was induced as described above, with IPTG-saturated nitrocellulose filters. These filters were then treated as for immunoperoxidase staining, but after incubation of the BP serum (diluted 1:100 in $3 \% \mathrm{BSA}$ in $0.5 \mathrm{M} \mathrm{NaCl}, 1 \% \mathrm{NP}-40,0.5 \%$ sodium deoxycholate in 10 $\mathrm{mM}$ Tris- $\mathrm{HCl}, \mathrm{pH} 7.4$ [WB2]), the filters, in $150-\mathrm{mm}$ culture dishes, were washed five times with WB2 then twice briefly with $\mathrm{H}_{2} \mathrm{O}$. The bound antibodies were eluted twice with $10 \mathrm{ml}$ of a $37^{\circ} \mathrm{C}$ solution of 50 $\mathrm{mM}$ glycine and $500 \mathrm{mM} \mathrm{NaCl}, \mathrm{pH} 2.3$, for 15 min each on a rocking platform. The eluates were immediately neutralized with $1 / 10 \mathrm{vol}$ of $500 \mathrm{mM} \mathrm{Na} 2 \mathrm{PO}_{4}$. The eluted, neutralized antibodies were dialyzed against PBS at $4^{\circ} \mathrm{C}$, then concentrated on a PM-10 filter (Amicon Corp., Danvers, MA) followed by a Centricon 30 (Amicon Corp.) to a final volume of $\sim 60-100 \mu \mathrm{l}$.

Restriction enzyme analysis of the $\lambda g t 11$ cDNA insert. A liquid lysate of the BP $\lambda$ gt 11 clone was prepared in Y 1088 cells. Phage DNA was isolated by a rapid lambda DNA extraction technique (21). In brief, cellular DNA, RNA, and some protein were removed from the lysate with DE52 cellulose (Whatman Laboratory Products, Inc., Hillsboro, OR) in LB broth. The phage protein and excess cellular proteins were then extracted with phenol/chloroform, leaving the phage DNA in the aqueous phase. After repeat extraction with chloroform, and ethanol precipitation, the phage DNA was digested with various restriction enzymes according to the manufacturer's directions (Bethesda Research Laboratories, Gaithersburg, MD). 5\% polyacrylamide and $0.8 \%$ agarose gels were used to identify digested fragments (18). A DNA 1-kb ladder (Bethesda Research Laboratories) was used as a standard.

$R N A$ slot blots and Northern blots. For slot blots $\sim 7 \mu \mathrm{g}$ of keratinocyte total RNA in $5 \mathrm{M}$ formaldehyde/7.5× standard saline citrate (SSC) $\left(20 \times \mathrm{SSC}=3 \mathrm{M} \mathrm{NaCl}, 0.3 \mathrm{M} \mathrm{Na}_{3}\right.$ citrate, $\left.\mathrm{pH} 7\right)$ was added per slot (Schleicher \& Schuell, Inc., Keene, NH) over nitrocellulose paper. For Northerns, $35 \mu \mathrm{g}$ of keratinocyte total RNA/lane was electrophoresed in formaldehyde/agarose gels, then blotted to nitrocellulose (18). An RNA ladder (Bethesda Research Laboratories) was used as a standard. RNA on nitrocellulose filters was prehybridized at $42^{\circ} \mathrm{C}$ with $50 \%$ formamide, $5 \times$ SSC, $2.5 \times$ Denhardt's solution, $0.1 \%$ SDS, 0.5 $\mathrm{mg} / \mathrm{ml}$ yeast tRNA in $20 \mathrm{mM}$ sodium phosphate buffer, $\mathrm{pH} 7$ (hybridization buffer). ${ }^{32} \mathrm{P}$-riboprobes for hybridization were prepared using either the T7 or SP6 promoter of the pGEM3 vector (Promega Biotech, Madison, WI), into which the Eco RI fragments of the cDNA from the BP $\lambda g t 11$ clone were subcloned. About $4 \times 10^{7} \mathrm{dpm}$ of each riboprobe in hybridization buffer were incubated at $55^{\circ} \mathrm{C}$ overnight on each slot blot or Northern strip. Nitrocellulose filters were then washed four times with $2 \times$ SSC and $0.1 \%$ SDS at room temperature, then twice with $0.1 \times$ SSC and $0.1 \%$ SDS at $68^{\circ} \mathrm{C}$. Filters were additionally treated with RNase A (Boehringer Mannheim Biochemicals, Indianapolis, IN), $300 \mu \mathrm{g} / \mathrm{ml}$, and RNase $\mathrm{T}_{1}$ (Boehringer-Mannheim Biochemicals) $3 \mu \mathrm{g} / \mathrm{ml}$ in $2 \times \mathrm{SSC}$ at $37^{\circ} \mathrm{C}$ for $30 \mathrm{~min}$ to digest single-stranded RNA, and washed once with $0.1 \times \mathrm{SSC}, 0.1 \%$ SDS at $68^{\circ} \mathrm{C}$. Filters were exposed to Kodak X-Omat AR film on an intensifying screen.

DNA sequencing. Double-stranded DNA inserts in pGEM3 were sequenced using the dideoxy chain termination method with a GemSeq K/RT kit according to the manufacturer's instructions (Promega Biotech). The cDNA was sequenced from both ends by use of the T7 and SP6 promoter primers and subsequently by synthetic 17-mer oligonucleotides made to previously sequenced regions. AMV reverse transcriptase as well as Klenow DNA polymerase was used. $\left[{ }^{35} \mathrm{~S}\right]-$ dATP-labeled DNA (Dupont New England Nuclear) was electrophoresed in 6 and $8 \%$ polyacrylamide-urea gels. 


\section{Results}

General characteristics of the $\lambda$ gt11 cDNA library. Poly $\left(\mathrm{A}^{+}\right)$ RNA isolated from cultured human keratinocytes, known to express the 230-kD BP antigen (10), was used to construct cDNA that was cloned into the Eco RI site of the $\lambda \mathrm{gt} 11$ expression vector (16). The poly $\left(\mathrm{A}^{+}\right) \mathrm{RNA}$ was primed with oligo-dT, thereby biasing the library to the $3^{\prime}$ end of the RNA (or to the C-terminal end of the corresponding protein). These cloned cDNAs should reflect sequences of the keratinocyte mRNAs, including the BP antigen. To determine the number of independent cDNA recombinants in the library, we titered the unamplified library on X-gal agar plates, on which nonrecombinant phages are blue and recombinant phages are white. The library contained $70 \%$ recombinants for a total of 2.7 $\times 10^{7}$ independent recombinants. As discussed in Methods, the average insert size was determined to be $1 \mathrm{~kb}$ (range $0.5-$ $3.8 \mathrm{~kb})$.

Because cDNAs containing coding sequences for keratins, the major proteins of keratinocytes, should be relatively abundant, we first tested the ability of this library to produce proteins immunoreactive with antibodies to keratin. We used antibodies raised to, and affinity-purified with, the $\mathrm{COOH}$-terminal end of K14 (22). We screened one $150-\mathrm{mm}$ plate of $\sim 10,000$ plaques with this anti-K14 antibody. At least 18 plaques stained clearly positive, suggesting the production of many fusion proteins with $\mathrm{K} 14$ sequences. One of these clones was further characterized and confirmed to be a keratin clone (data not shown). These results suggest that coding sequences for abundant proteins are easily detectable in our library. Since $1 \times 10^{6}$ clones can practically be screened by this method, even proteins expressed at $1 / 100$ to $1 / 1,000$ the level of K14 should be detectable. Therefore, we used this library to try to find a clone containing a cDNA with coding sequences for BP antigen, which is presumably expressed at much lower levels than K14.

Identification of a $\lambda$ gt $11 \mathrm{cDNA}$ clone with coding sequences for BP antigen. Plaques from another 30,000 clones in the library were screened by the immunoperoxidase technique with one particular BP serum. This serum stained three clones. Only the darkest staining clone, called here the BP clone, was further characterized. To determine whether the protein produced by this putative BP clone specifically bound antibodies in BP sera, we partially purified the clone so that the immunoperoxidase staining of this clone with many different sera could be compared with the background (nonspecific) staining of other clones. Of 9 BP sera, all specifically stained the plaques of this IPTG-induced clone, whereas 6 normal sera and 11 pemphigus disease control sera did not (Fig. 1). These results suggest that this $\mathrm{BP}$ clone encodes a major antigenic site of the BP antigen.

To further confirm that the antibodies binding to the products of this clone were typical BP antibodies in that they were capable of binding to the epidermal BMZ and they could immunoprecipitate the BP antigen, we affinity-purified the IgG that bound the purified BP clone. The BP clone was recloned until it was pure and then incubated with the BP serum. After extensive washing, bound BP IgG was eluted with acid glycine (Fig. $2 A$ ). This affinity-purified IgG was shown by immunofluorescence to bind the BMZ of epidermis (Fig. $2 B$ ). In addition, the affinity-purified IgG immunoprecipitated the $230-\mathrm{kD}$ BP antigen extracted from metabolically radiolabeled cultured keratinocytes (Fig. $2 C$ ). These results confirm that the cDNA insert of the BP $\lambda$ gt 11 clone contains coding sequences for a major antigenic site of BP antigen.

Eco RI digestion of the $\lambda \mathrm{gt} 11 \mathrm{BP}$ clone revealed a cDNA insert of two fragments of $\sim 680$ and $1,500 \mathrm{bp}$, as determined by polyacrylamide and agarose gel electrophoresis.

Northern analysis of keratinocyte $R N A$ for $B P m R N A$. To determine the direction of transcription to make antisense riboprobes and to determine the $5^{\prime}$ end (in relation to sense mRNA transcription) of the 680- and 1,500-bp Eco RI fragments, we subcloned them into the pGEM3 vector. Two clones were found in which the 1,500-bp fragment was in opposite orientation (BP1500A, BP1500B), as determined by restriction enzyme analysis. ${ }^{32} \mathrm{P}$-riboprobes from each were made with the T7 promoter. Slot blots on keratinocyte total RNA indicated that the BP1500B clone produced an antisense (complementary) probe to the BP mRNA (Fig. 3). ${ }^{32} \mathrm{P}$-riboprobes were made in both directions from the 680-bp fragment by using the T7 and SP6 promoter. Transcription from the

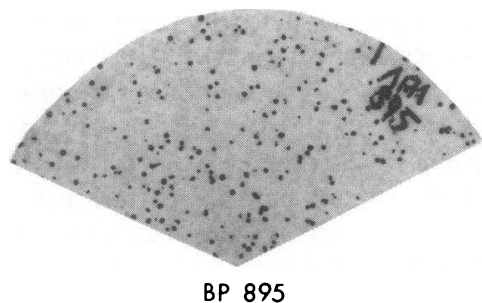

BP 895

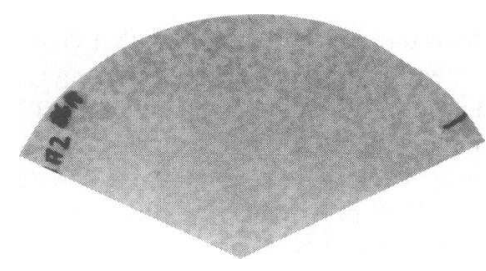

PV 86

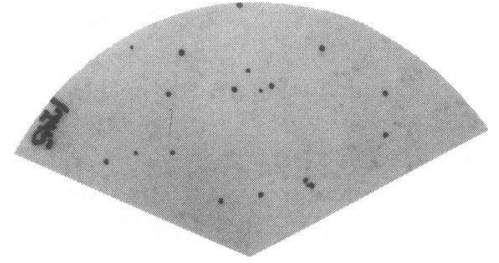

BP 245

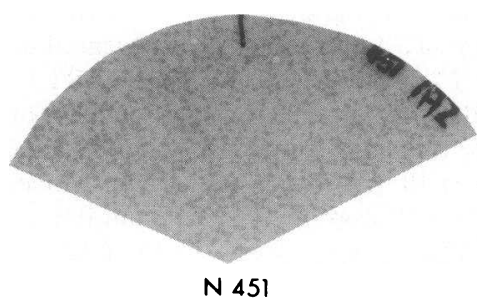

N 451
Figure 1. Immunoperoxidase staining of plaques of the partially purified $\lambda \mathrm{gt} 11 \mathrm{BP}$ clone on nitrocellulose filters demonstrates specific staining with BP sera. Note the dark staining of the BP clone plaques compared with the faint nonspecific staining of the background plaques. The normal human $(\mathrm{N})$ and pemphigus vulgaris (PV) control sera do not stain the BP clone plaques. 

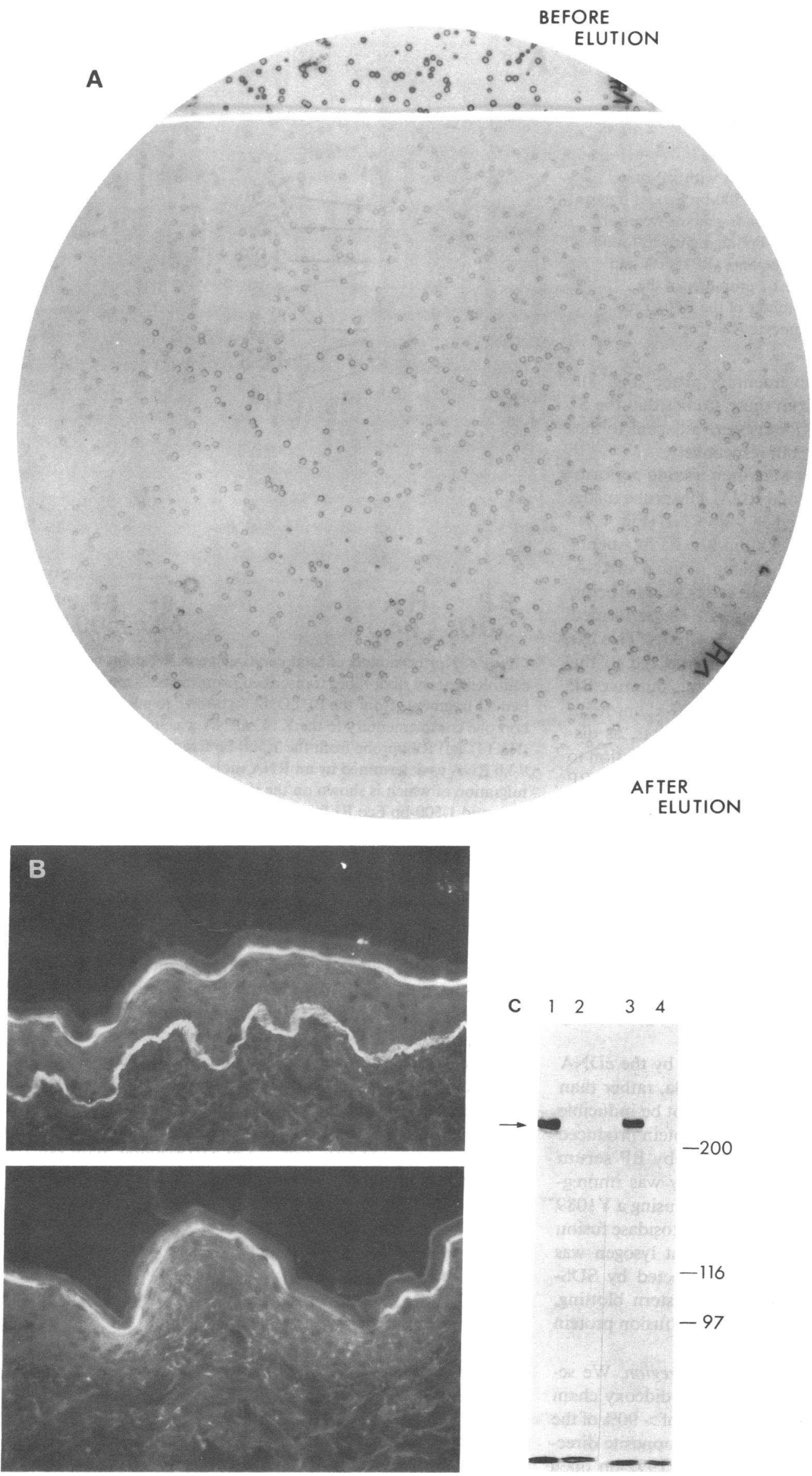

Figure 2. BP IgG affinity-purified on plaques of the $\lambda g t 11$ BP clone stains the epidermal BMZ by immunofluorescence and immunoprecipitates the $230-\mathrm{kD}$ BP antigen. $(A)$ Immunoperoxidase staining of plaques of the purified BP clone on a nitrocellulose filter. The filter was incubated with BP serum then washed. A small piece of the top of the filter (before elution) was cut off and stained. The bound BP IgG on the rest of the filter (after elution) was eluted with acid glycine, then similarly stained. Much of the affinity-purified IgG was eluted, accounting for the less intense staining after elution. (B) Indirect immunofluorescence on normal skin with the BP IgG affinity-purified on the plaques of the BP clone shows staining of the epidermal BMZ (top). IgG from the same BP serum similarly eluted from plaques of control $\lambda g t 11$ clones did not demonstrate this staining (bottom). (C) SDS-PAGE of immunoprecipitations of ${ }^{14} \mathrm{C}$-amino acid-labeled proteins extracted from cultured keratinocytes demonstrates the 230-kD BP antigen (arrow) precipitated by BP serum (lane $I$ ) and by BP IgG affinity purified on plaques of the BP clone (lane 3), but not by normal human serum (lane 2) or IgG affinity purified from BP serum on plaques of control clones (lane 4). Migration of molecular weight standards in $\mathrm{KD}$ is indicated on the right. 


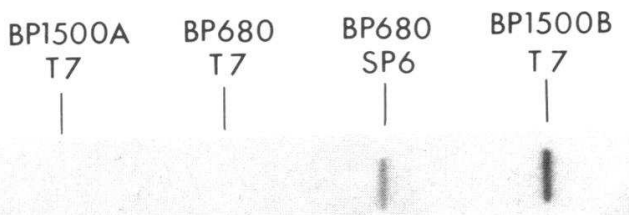

Figure 3. RNA slot blot of total keratinocyte RNA with ${ }^{32} \mathrm{P}$-riboprobes made by transcription of the 680 - and 1,500 -bp Eco RI fragments of the cDNA insert in the BP clone. The fragments were subcloned into the pGEM3 plasmid. The 1,500-bp fragment was found in opposite orientations in two different subclones (BP1500A and BP1500B). The riboprobes made with the T7 promoter of the BP1500B subclone and with the SP6 promoter of the BP680 subclone were complementary to the keratinocyte RNA.

SP6 promoter produced the complementary probe (Fig. 3). Conversely, we could determine from these studies that the $5^{\prime}$ ends of the 1,500- and 680-bp fragments were adjacent to the T7 promoter in BP1500A and BP680, respectively.

The complementary riboprobes were then used to perform Northern analysis on keratinocyte total RNA. The probe made from the 1,500-bp fragment identified 9-kb RNA (Fig. $4 A$ ). To confirm that both the 680- and 1,500-bp Eco RI fragments in the $\lambda \mathrm{gt} 11 \mathrm{BP}$ clone came from the same original mRNA (rather than coming from two different mRNAs and being cloned in tandem into the Eco RI cloning site), we performed Northern blotting with probes from both fragments. This experiment demonstrated that both probes hybridized to the 9-kb RNA as expected (Fig. $4 B$ ). The size of this putative BP mRNA is easily large enough to encode for a $230-\mathrm{kD}$ protein.

Orientation of the BP cDNA in $\lambda g t 11$. Slot blots, as discussed above, were used to determine the $5^{\prime}$ ends, in relation to sense mRNA transcription, of the 1,500- and 680-bp BP cDNA Eco RI fragments. Restriction enzyme analysis with $\mathrm{Xba} I$ of these fragments in plasmid vector (pGEM; Promega Biotec, Madison, WI) revealed that there were Xba I sites on the $5^{\prime}$ end of the 1,500-bp fragment but no sites in the 680-bp fragment (Fig. 5). DNA sequencing of both fragments confirmed these findings. An Xba I, Kpn I double digest of the $\lambda \mathrm{gt} 11 \mathrm{BP}$ clone revealed that the cDNA insert was oriented so that its direction of transcription ( $5^{\prime}$ end) was opposite to that of the lac $\mathrm{Z}$ gene (Fig. 5). This direction of orientation has been previously reported for cDNAs cloned by antibody screening of $\lambda \mathrm{gt} 11(23,24)$.

Since, presumably, the peptide encoded for by the cDNA in this orientation is promoted by a late lambda, rather than the lac $Z$, promoter (23), its synthesis should not be inducible by IPTG. Indeed, this is what we found: the protein produced by plaques from this BP clone was stained by BP serum whether or not the nitrocellulose filter overlay was impregnated with IPTG (data not shown). In addition, using a Y1089 lysogen (20) we were unable to induce a $\beta$-galactosidase fusion protein with the BP clone. A nonrecombinant lysogen was induced to synthesize $\beta$-galactosidase as detected by SDSPAGE with Coomassie Blue staining and Western blotting, but the BP clone lysogen did not demonstrate a fusion protein by either of these techniques.

$D N A$ sequencing of the $B P$ cDNA coding region. We sequenced the BP cDNA from its 5 ' end by the dideoxy chain termination method. In addition, the sequence of $>90 \%$ of the cDNA was confirmed by sequencing from the opposite direction. In one of three frames we found a long 1992-bp open
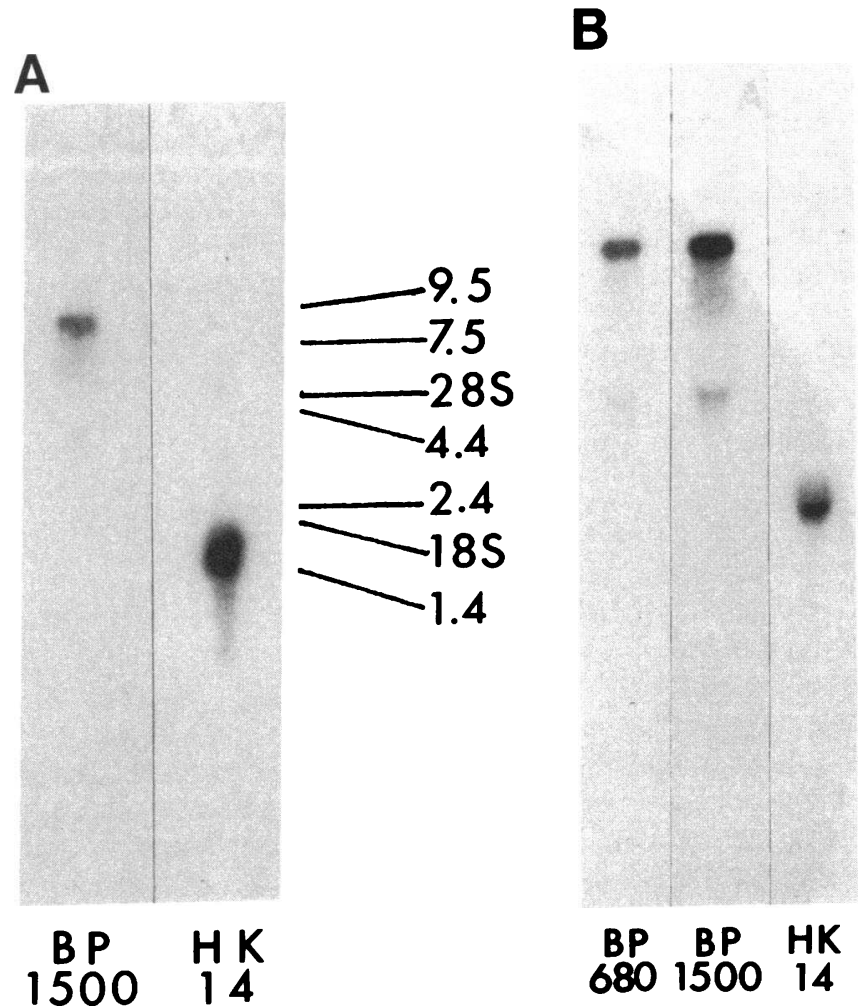

Figure 4. Northern blots of total keratinocyte RNA demonstrate that complementary riboprobes transcribed from the 680- and 1,500-bp Eco RI fragments from the BP cDNA hybridize to a 9-kb RNA. A riboprobe complementary to the K14 mRNA was used as a control (HK14). (A) Riboprobe from the 1,500-bp fragment hybridizes to a 9-kb RNA as determined by an RNA molecular weight ladder, the migration of which is shown on the right. $(B)$ Probes from both the 680- and 1,500-bp Eco RI fragments hybridize to comigrating 9-kb RNA.

reading frame (ORF), whereas the other two frames showed multiple stop codons. The deduced amino acid sequence of the long ORF is shown in Fig. 6. The base sequence of the DNA containing this long ORF did not have homology to known sequences, as determined by a computer search for homology with the primate, rodent, other mammals, other vertebrates, and viral or unannotated DNA sequences stored in GenBank. In addition, the amino acid sequence of the deduced peptide did not show striking homology with any sequences in the National Biomedical Research Foundation protein bank. The best homology was only $18 \%, 74$ of 410 residues, with myosin alpha heavy chain from rabbit cardiac muscle.

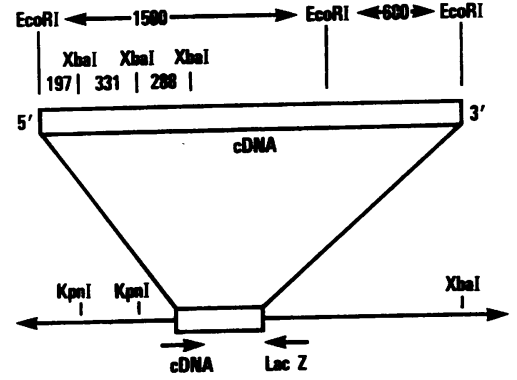

Figure 5. Scheme of the cDNA insert in the $\lambda g t 11$ BP clone. The arrows indicate the direction of transcription of the lac $\mathrm{Z}$ and the cDNA. The restriction sites shown were used to orient the cDNA within $\mathrm{gt} 11$. 
GAAT TACACT TAAAGACCATAGAGGAGCAGATGACCCACAGAAAGATGGT TCTGT T TCAGGAAGAATCTGGTAAAT TCAAACAATCAGCAGAGGAG

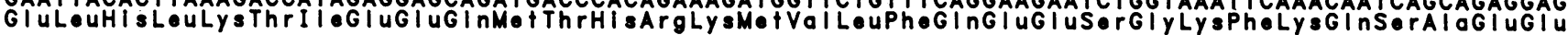
IT TCGGAAGAAGATGGAAAAAT TAATGGAGTCCAAAGTCATCACTGAAAATGATATT TCAGGCATTAGGCT TGACT T TGTGTCTCT TCAACAAGAA

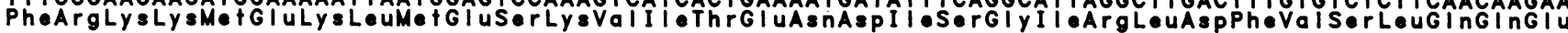
AACTCTAGAGCCCAAGAAAATGCTAAGCT T TGTGAAACAAACAT TAAAGAACT TGAAAGACAGCT TCAACAGTATCGTGAACAAATGCAGCAAGGG

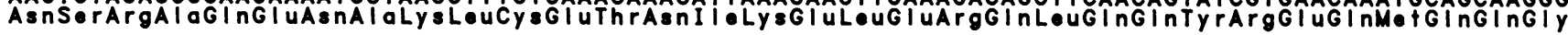
CAGCACATGGAAGCAAATCAT TACCAAAAATGTCAGAAACT TGAGGATGAGCTGATAGCCCAGAAGCGTGAGGT TGAAAACCTGAAGCAAAAAATG GI GACCAACAGATCAAAGAGCATGAACATCAATTAGTTTTGCTCCAGTGTGAAATTCAAAAAAAGAGCACAGCCAAAGACTGTACCTTCAAACCAGAT

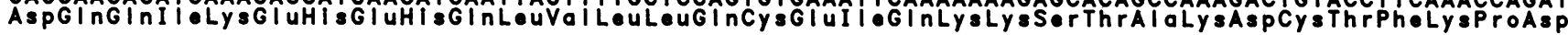
TT TGAGATGACAGTGAAGGAGTGCCAGCACTCTGGAGAGCTGTCCTCTAGAAACACTGGACACCT TCACCCAACACCCAGATCCCCTCTGTTGAGA

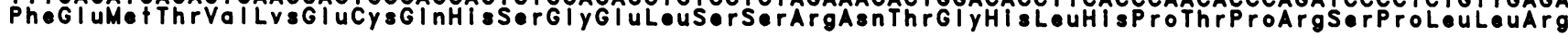
TGGACTCAAGAACCACAGCCATTGGAAGAGAAGTGGCAGCATCGGGTTGT TGAACAGATACCCAAAGAAGTCCAATTCCAGCCACCAGGGGCTCCA TrPTh CTCGAGAAAGAGAAAAGCCAGCAGTGT TACTCTGAGTACTTTTCTCAGACAAGCACCGAGTTACAGATAACT T TGATGAGACAAACCCCATTACA

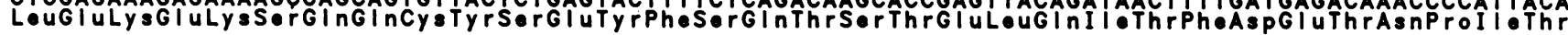
AGACTGTCTGAAATTGAGAAGATAAGAGACCAAGCCCTGAACAATTCTAGACCACCTGTTAGGTATCAAGATAACGCATGTGAAATGGAACTGGTG

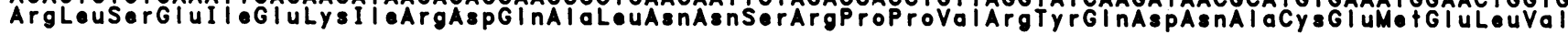
AAGGT TT TGACACCCT TAGAGATAGCTAAGAACAAGCAGTATGATATGCATACAGAAGTCACAACATTAAAACAAGAAAAGAACCCAGT TCCCAGT

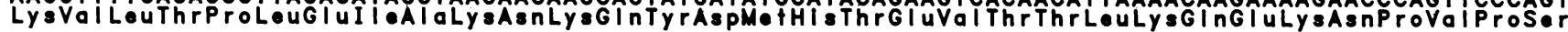
GCTGAAGAATGGATGCTTGAAGGGTGCAGAGCATCTGGTGGACTCAAGAAAGGGGATT TCCT TAAGAAGGGCTTAGAACCAGAGACCTTCCAGAAC

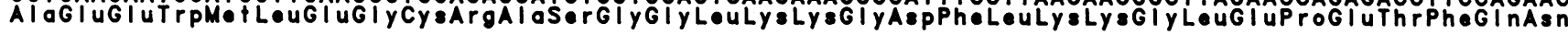
TT TGATGGTGATCATGCATGT TCAGTCAGGGATGATGAAT T TAAAT TCCAAGGGCT TAGGCACACTGTGACTGCCAGGCAGT TGGTGGAAGCTAAG

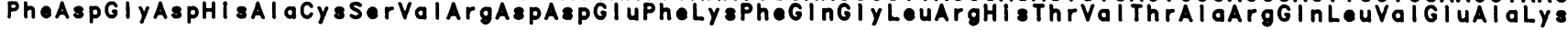
CTTCTGGACATGAGAACAAT TGAGCAGCTGCGACTCGGTCTTAAGACTGTTGAAGAAGTTCAGAAAACTCT TAACAAGTT TCTGACGAAAGCCACC

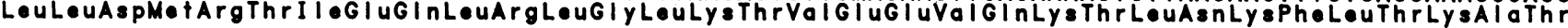
TCAATTGCAGGGCTTTACCTAGAATCTACAAAAGAAAAGATT TCATTTGCCTCAGCGGCCGAGAGAATCATAATAGACAAAATGGTGGCTTTGGCA

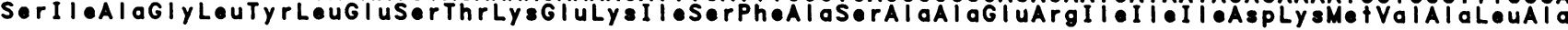
TT TT TAGAAGCTCAGGCTGCAACAGGT TTTATAATTGATCCCATTTCAGGTCAGACATATTCTGTTGAAGATGCAGTTCTTAAAGGAGTTGTTGAC

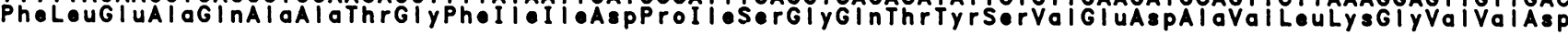
CCCGAAT TCAGAAT I AGGCT TCT TGAGGCAGAGAAGGCAGCTGTGGGATAT TCT TAT TCT TCTAAGACAT TGTCAGTGTT TCAAGCTATGGAAAAT

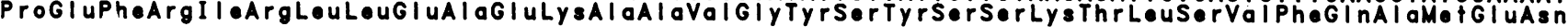
AGAATGCTTGACAGACAAAAAGGTAAACATATCTTGGAAGCCCAGATTGCCAGTGGGGGTGTCATTGACCCTGTGAGAGGCATTCGTGTTCCTCCA

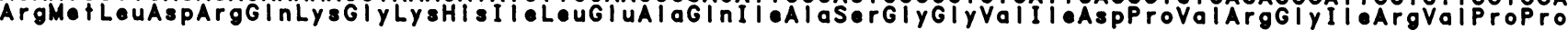
GAAATTGCTCTGCAGCAGGGGTTGT TGAATAATGCCATCT TACAGT T T T TACATGAGCCATCCAGCAACACAAGAGT T TCCCTAATCCCAATAAC

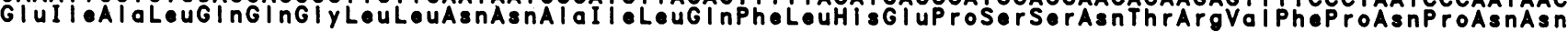
AAGCAAGCTCTGTATTACTCAGAATTACTGCGAATGTGTGTATT TGATGTAGAGTCCCAATGCTT TCTGTT TCCATT TGGGGAGAGGAACATT TCC

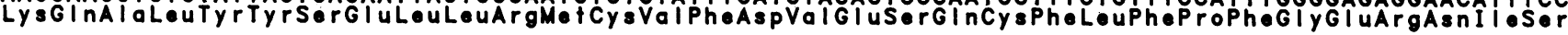
AATCTCAATGTCAAGAAAACACATAGAATT TCTGTAGTAGATACTAAAACAGGATCAGAATTGACCGTGTATGAGGCTT TCCAGAGAAACCTGAT

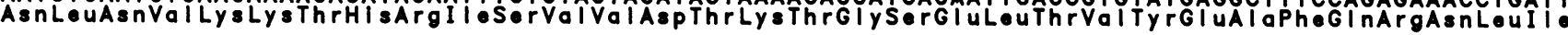
GAGAAAAGTATATATCTTGAACTTTCAGGGCCTTTTTGGGATACAGGATTTTCTTTATTCAGGTCAAAAAAATGA

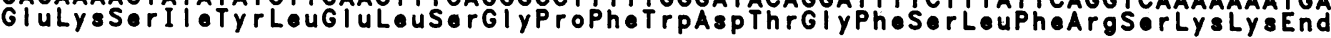

Figure 6. ORF and deduced amino acid sequence of the BP cDNA.

\section{Discussion}

Autoantibodies from BP patients were used to isolate a unique cDNA clone encoding major antigenic determinants of the BP antigen. Of the nine BP sera tested, all bound antigenic sites produced by this BP clone but not other, either recombinant or nonrecombinant, $\lambda \mathrm{gt} 11$ clones. In addition, antibodies affinity-purified on the products of this BP clone bound the epidermal BMZ by immunofluorescence and immunoprecipitated the $230-\mathrm{kD}$ BP antigen.

The $\lambda \mathrm{gt} 11$ expression vector was designed to use the lac promoter to express the peptide encoded for by the cDNA as a fusion protein with $\beta$-galactosidase. However, inserted cDNA that is capable of producing immunoreactive protein may be oriented so that its direction of transcription is opposite to that of the lac $\mathrm{Z}$ gene $(23,24)$. In this case the expression of the protein encoded for by the cDNA is presumably from a late phage lambda promoter (23). The BP cDNA was similarly oriented. This finding was consistent with the fact that IPTG was not needed to induce expression of protein immunoreactive with BP serum.

Northern analysis with the BP cDNA probe indicated that the mRNA encoding for BP antigen is $9 \mathrm{~kb}$. Since $\sim 6.5 \mathrm{~kb}$ of coding mRNA is necessary to encode for $\sim 230-\mathrm{kD}$ polypeptide, the large size of the BP mRNA implies that: (a) BP antigen is synthesized as a larger precursor, and/or $(b)$ there is a long $3^{\prime}$ and/or $5^{\prime}$ untranslated region of the BP mRNA. Since we did not detect the poly $A$ tail or polyadenylation signal in the BP cDNA clone (data not shown), we cannot predict the length of the $5^{\prime}$ end of the mRNA.

The long ORF of the BP cDNA would encode a polypeptide of about 76,000 D, one-third of the full length of the 230-kD BP antigen. Since the BP cDNA contains the stop codon at the end of this long ORF, it reflects sequences from the $3^{\prime}$ end of the mRNA, consistent with the construction of the $\lambda g t 11$ library with an oligo-dT primer. We can conclude from the above data that the $\mathrm{COOH}$-terminal end of the BP antigen contains major antigenic sites to which most, if not all, BP antibodies bind.

This study, as well as others (25-29), has demonstrated that autoantibodies from patients can be used to isolate cDNA clones for the corresponding antigens from $\lambda g t 11$ expression libraries. This powerful technique should provide a means for the characterization of these antigens at a protein and genetic level. Thus, in the case of BP antigen, the cDNA probe described in this paper and its sequence should prove useful in 
isolating a full-length cDNA clone (and the corresponding amino acid sequence for the whole antigen). This will in turn provide valuable information about hemidesmosomes, of which BP antigen is a component (4-6). In addition, because a probe for BP antigen is now available, studies on the BP genomic DNA and the genetic regulation of expression of BP antigen in differentiating epidermis are feasible. Since BP antigen is produced by basal cells but not more differentiated keratinocytes (8), these studies should shed light on control of epidermal differentiation.

Finally, this technique of autoantibody screening of cDNA expression libraries would also be feasible for isolation of cDNA clones for other autoimmune blistering diseases such as pemphigus and epidermolysis bullosa acquisita. The autoantibodies in these diseases, as well as in BP, define molecules that are tissue-specific for stratified squamous epithelia, such as skin and mucous membrane. Like BP antigen, these molecules too are regulated with differentiation of epidermis (8). The isolation of these cDNA probes will undoubtedly provide valuable tools for our understanding of normal and diseased skin and mucous membranes.

\section{Acknowledgments}

We thank Dr. Peter Howley and Dr. Douglas Lowy for their invaluable discussions; Drs. William Phelps, Pamela Hawley-Nelson, and Thomas Kreig for their advice and technical assistance; and Dr. Stephen Katz for review of the manuscript.

\section{References}

1. Beutner, E. H., R. E. Jordon, and T. P. Chorzelski. 1968. The immunopathology of pemphigus and bullous pemphigoid. J. Invest. Dermatol. 51:63-80.

2. Sams, W. M., and W. R. Gammon. 1982. Mechanism of lesion production in pemphigus and pemphigoid. J. Am. Acad. Dermatol. 6:431-449.

3. Diaz, L. A., H. J. Weiss, and N. J. Calvanico. 1978. Phylogenetic studies with pemphigus and pemphigoid antibodies. Acta Dermato-Venereol. 58:537-540.

4. Mutasim, D. F., Y. Takahashi, R. S. Labib, G. J. Anhalt, H. P. Patel, and L. A. Diaz. 1985. A pool of bullous pemphigoid antigen is intracellular and associated with the basal cell cytoskeleton-hemidesmosome complex. J. Invest. Dermatol. 84:47-53.

5. Westgate, G. E., A. C. Weaver, and J. R. Couchman. 1985. Bullous pemphigoid antigen localization suggests an intracellular association with hemidesmosomes. J. Invest. Dermatol. 84:218-224.

6. Regnier, M., P. Vaigot, S. Michel, and M. Prunieras. 1985. Localization of bullous pemphigoid antigen in isolated human keratinocytes. J. Invest. Dermatol. 85:187-190.

7. Stanley, J. R., J. M. Foidart, J. C. Murray, G. R. Martin, and S. I. Katz. 1980. The epidermal cell which selectively attaches to a collagen substrate is the basal cell. J. Invest. Dermatol. 74:54-58.

8. Stanley, J. R., and S. H. Yuspa. 1983. Specific epidermal protein markers are modulated during calcium-induced terminal differentiation. J. Cell Biol. 96:1809-1814.

9. Tidman, M. J., and R. A. J. Eady. 1986. Hemidesmosome heterogeneity in junctional epidermolysis bullosa revealed by morphometric analysis. J. Invest. Dermatol. 86:51-56.

10. Stanley, J. R., P. Hawley-Nelson, S. H. Yuspa, E. M. Shevach, and S. I. Katz. 1981. Characterization of bullous pemphigoid antigen: a unique basement membrane protein of stratified squamous epithelia. Cell. 24:897-903.
11. Stanley, J. R., D. T. Woodley, and S. I. Katz. 1984. Identification and partial characterization of pemphigoid antigen extracted from normal human skin. J. Invest. Dermatol. 82:108-111.

12. Stanley, J. R. 1985. A specific antigen-antibody interaction triggers the cellular pathophysiology of bullous pemphigoid. $B r . J$. Dermatol. 113(Suppl. 28):67-73.

13. Labib, R. S., G. J. Anhalt, H. P. Patel, D. F. Mutasim, and L. A. Diaz. 1986. Molecular heterogeneity of the bullous pemphigoid antigens as detected by immunoblotting. J. Immunol. 136:1231-1235.

14. Hintner, J., P. M. Steinert, and T. J. Lawley. 1983. Human upper epidermal cytoplasmic antibodies are directed against keratin intermediate filament proteins. J. Clin. Invest. 71:1344-1351.

15. Thivolet, C. H., H. H. Hintner, and J. R. Stanley. 1984. The effect of retinoic acid on the expression of pemphigus and pemphigoid antigens in cultured human keratinocytes. J. Invest. Dermatol. 82:329-334.

16. Young, R. A., and R. W. Davis. 1983. Efficient isolation of genes by using antibody probes. Proc. Natl. Acad. Sci. USA. 80:11941198.

17. Maniatis, T., E. F. Fritsch, and J. Sambrook. 1982. Molecular Cloning: A Laboratory Manual. Cold Spring Harbor Laboratory, Cold Spring Harbor, NY. 545 pp.

18. Davis, L. G., M. D. Dibner, and J. F. Battey. 1986. Basic Methods in Molecular Biology. Elsevier, New York. 388 pp.

19. Gubler, V., and B. J. Hoffman. 1983. A simple and very efficient method for generating cDNA libraries. Gene (Amst.). 25:263269.

20. Huynh, T. V., R. A. Young, and R. W. Davis. 1985. Constructing and screening cDNA libraries in $\lambda g t 10$ and $\lambda g t 11$. In DNA Cloning: A Practical Approach. D. M. Glover, editor. IRL Press, Oxford. 49-78.

21. Benson, S. A., and R. K. Taylor. 1984. A rapid small-scale procedure for isolation of phage lambda DNA. Biotechniques. 2:126127.

22. Roop, D. R., C. K. Cheng, R. Toftgard, J. R. Stanley, P. M. Steinert, and S. H. Yuspa. 1985. The use of cDNA clones and monospecific antibodies as probes to monitor keratin gene expression. Ann. NY Acad. Sci. 455:426-435.

23. Goto, T., and J. C. Wang. 1984. Yeast DNA topoisomerase II is encoded by a single-copy, essential gene. Cell. 36:1073-1080.

24. Dame, J. B., J. L. Williams, T. F. McCutchan, J. L. Weber, R. A. Wirtz, W. T. Hockmeyer, W. L. Maloy, J. D. Haynes, I. Schneider, D. Roberts, G. S. Sanders, P. Reddy, C. L. Diggs, and L. H. Miller. 1984. Structure of the gene encoding the immunodominant surface antigen on the sporozoite of the human malaria parasite Plasmodium falciparum. Science (Wash. DC). 225:593-599.

25. Chambers, J. C., and J. D. Keene. 1985. Isolation and analysis of cDNA clones expressing human lupus La antigen. Proc. Natl. Acad. Sci. USA. 82:2115-2119.

26. Habets, W. J., P. T. G. Sillekens, M. H. Hoet, J. A. Schalken, A. J. M. Roebroek, J. A. M. Leunissen, W. J. M. van de Ven, and W. J. van Venrooij. 1987. Analysis of a cDNA clone expressing a human autoimmune antigen: full-length sequence of the U2 small nuclear RNA-associated B" antigen. Proc. Natl. Acad. Sci. USA. 84:24212425.

27. Dropcho, E. J., Y. T. Chen, J. B. Posner, and L. J. Old. 1987. Cloning of a brain protein identified by autoantibodies from a patient with paraneoplastic cerebellar degeneration. Proc. Natl. Acad. Sci. USA. 84:4552-4556.

28. Query, C. C., and J. D. Keene. 1987. A human autoimmune protein associated with U1 RNA contains a region of homology that is cross-reactive with retroviral p30 antigen. Cell. 51:211-220.

29. Seto, H. H., R. P. Magnusson, S. Filetti, and B. Rapoport. 1987. Molecular cloning and partial characterization of a new autoimmune thyroid disease-related antigen. J. Clin. Endocrinol. \& Metab. 64:578584. 